\title{
Computer Simulation of The Inducing Effects on the Phase Morphology of PS-b- PMMA Copolymers
}

\author{
Jianquan Li \\ Photoelectric Engineering College \\ Zaozhuang University, \\ Zaozhuang, China, 277160 \\ huangxuri2005@yahoo.com.cn
}

\author{
Dan $\mathrm{Mu}$ \\ College of Chemistry Chemical Engineering and \\ Materials Science \\ Zaozhuang University, \\ Zaozhuang, China,277160
}

\begin{abstract}
The phase morphologies of three groups of designed polystyrene-block-poly(methyl methacrylate) (PS-b-PMMA) copolymers were studied at 383,413 and $443 \mathrm{~K}$ by mesoscopic simulations. Eighteen patterned surfaces of four series were designed and designated as "ci", "co", "gra" and "rg" to study their influence on changing the microscopic phase morphology of copolymers. The degree of phase separation depended on the structures of copolymers, the topography of surfaces and the simulation temperatures. When the triblock copolymer with surfaces induced was composed of the same component at both ends and had larger PS segment percentage, it would present higher values of order parameter, that was, "ABA" showed the highest, "AB" the second high and "BAB" the last, the values of order parameter of the long chains were higher than those of the short ones, except the situation with co-4432 and co-8832 surfaces induced. However, the co-4432 and co-8832 surfaces performed the most intensive extent of inducing effect on changing the phase morphology of PS-b-PMMA copolymers. Temperature was a less effective influencing factor, compared with the others; but it was an important role in the extent of changing the phase morphology.
\end{abstract}

Keywords--Inducing effect; PS-b-PMMA copolymer; phase morphology

\section{INTRODUCTION}

Block copolymers are macromolecules composed of sequences of chemically distinct and mutually incompatible sequence of reapeat units that are covalently bonded. They tend to form various ordered morphologies which are in general on the nanometer scale through self-assembly and microphase separation. Recently, a number of works have focused on the phase behavior of block copolymer and their intriguing properties in research areas, such as supramolecular chemistry, materials science, and nanotechnology[1-5]. Self-assembly of block copolymers depends on molecular weight, segment size, and the strength of interaction between the blocks, represented by the FloryHuggins interaction parameter, $\chi$. The morphology depends on $\chi$, the composition of the copolymers and the volume fraction of one of the constituent blocks[6,7]. Thus block copolymers have attracted many researchers' attention in both theoretical and application fields [8-10].

PS/PMMA binary blends are a well-known immiscible combination, and can be observed bulk and surface phase separation 30,31 . Polystyrene-block-poly(methyl methacrylate) (PS-b-PMMA) copolymer is a polymer composed of two immiscible polymers blocks, which was studied in our former work; we had obtained the representative chain lengths of PS and PMMA chain, the $\chi$ data of ten different compositions which can cover most compositions at 383, 413 and $443 \mathrm{~K}$, and these data could be applied as the input parameters to deal with the PS-b-PMMA copolymers in this work.

The mesoscopic simulations in this paper were all related to the surface inducing effects on the compatibility of PS-bPMMA copolymers. It mainly contained the calculations of the surface inducing effects on changing the phase morphology of the PS-b-PMMA copolymers at 383, 413 and $443 \mathrm{~K}$. Thus, our study results would be a strong proof from theoretical view.

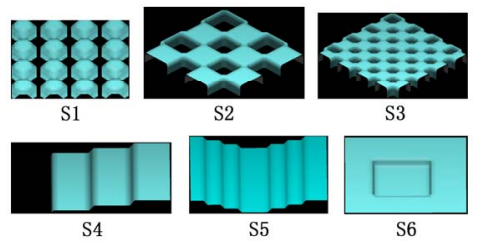

Figure 1. The scheme of six representative inducing surface

\section{MODELS AND SimUlATIONS}

To study the compatibility of different PS-b-PMMA copolymers, six types of copolymers denoted as A3B6, $\mathrm{A} 6 \mathrm{~B} 12, \mathrm{~A} 3 \mathrm{~B} 6 \mathrm{~A} 3$, A6B12A6, B6A3B6 and B12A6B12 were designed, in which $A$ and $B$ represented PS and PMMA segments, respectively. For convenience, A3B6 and A6B12 were defined as the "AB" groups; A3B6A3 and A6B12A6 were defined as the "ABA" group; B6A3B6 and B12A6B12 were defined as the "BAB" group. The former of each group represented short PS-b-PMMA copolymer chains and the latter represented long ones; the chain length of the latter was twice as long as the former in the same group. Six types of copolymer models were given in Table I in detail. The classified group information, the weight percentages of PMMA content and the input parameters of MesoDyn at 383, 413 and $443 \mathrm{~K} 32$ were also listed in this table.

We designed four series patterned surfaces as substrates, designated as "ci", "co", "gra" and "rg" series, to study its inducing effects on the compatibility of PS-b-PMMA copolymers. The number of each series was two, eight, five and three, respectively, named as ci-444, ci-882; co-444, co448, co-4412, co-4432, со-884, со-888, со-8812, со-8832; 
gra-444, gra-888, gra-2(444), gra-2(448), gra-2(888); rg-442, rg-884 and rg-16168. According to the characteristic of these eighteen surfaces, they could be classified into six types, which were "ci-xxx", "co-4xx", "co-8xx", "gra-xxx", "gra$2(\mathrm{xxx})$ " and "rg-xxx". The details about these six types of designed surfaces were listed in Table II, and six representative surfaces (ci-444, co-444, co-884, gra-444, gra2(444) and rg-442) were showed in Figure 1 as schemes (S1, S2, S3, S4, S5 and S6, respectively).
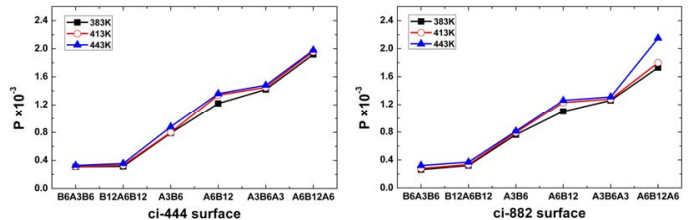

Figure 2. The order parameter values of PS-b-PMMA copolymers with ci-444 and ci-882 surface induced at 383,413 and $443 \mathrm{~K}$
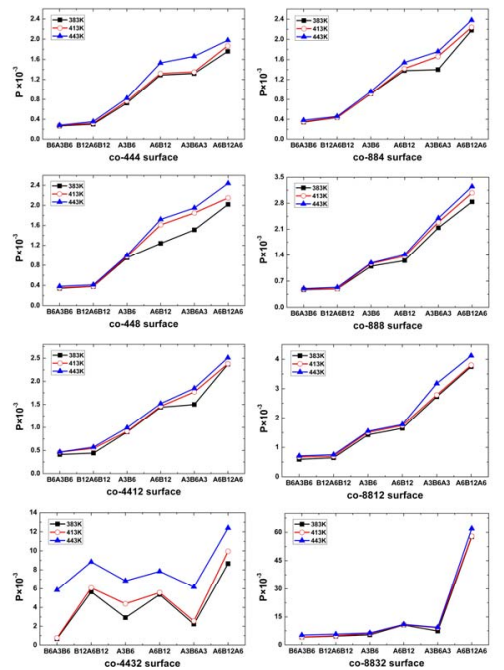

Figure 3. The order parameter values of PS-b-PMMA copolymers with co-444, co-448, co-4412, co-4432, co-884, co-888, co-8812 and co-8832 surface induced at 383,413 and $443 \mathrm{~K}$
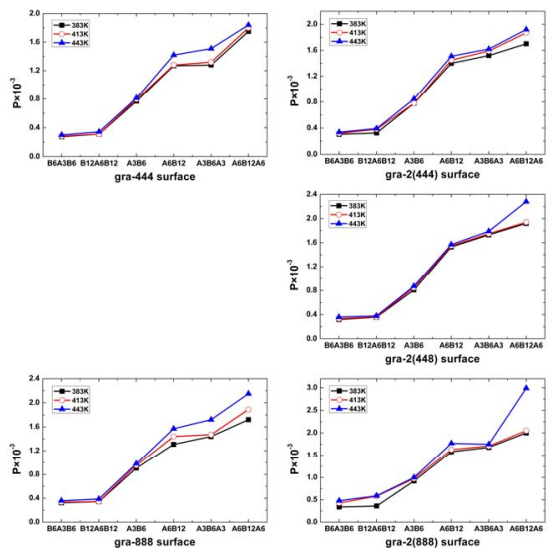

Figure 4. The P values of PS-b-PMMA copolymers with gra-444, gra-888, gra-2(444), gra-2(448) and gra-2(888) surface induced at 383, 413 and 443

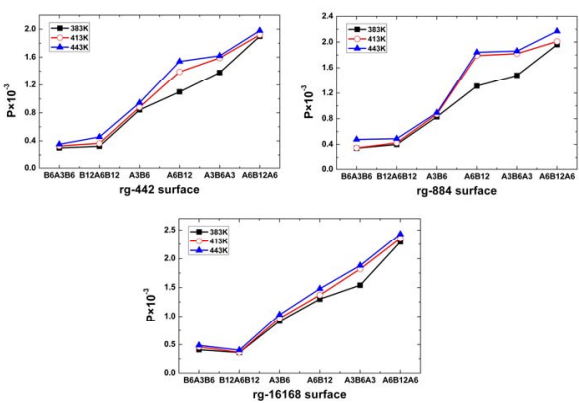

Figure 5. The order parameter values of PS-b-PMMA copolymers with rg-442, rg-884 and rg-16168 surface induced at 383, 413 and 443

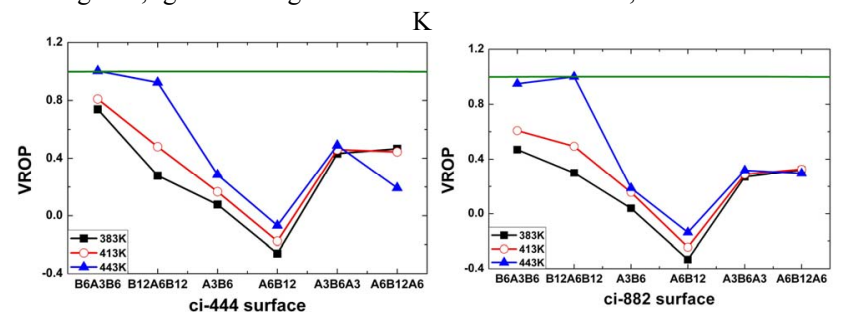

Figure 6. VROP data for PS-b-PMMA copolymers induced by ci-444 and ci-882 surface at 383,413 and 443
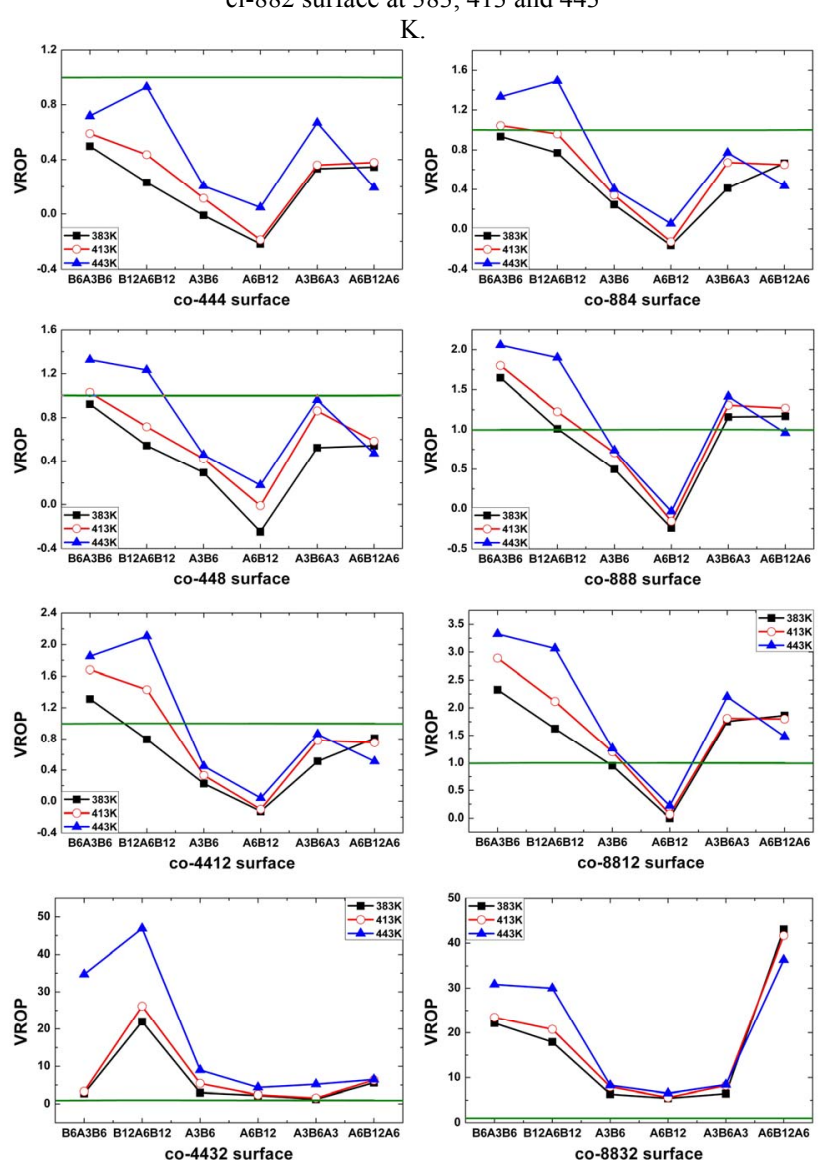

Figure 7. VROP data for PS-b-PMMA copolymers induced by co-444, co-448, co-4412, co-4432, co-884, co-888, co-8812 and co-8832 surface at 383,413 and 443 
K.
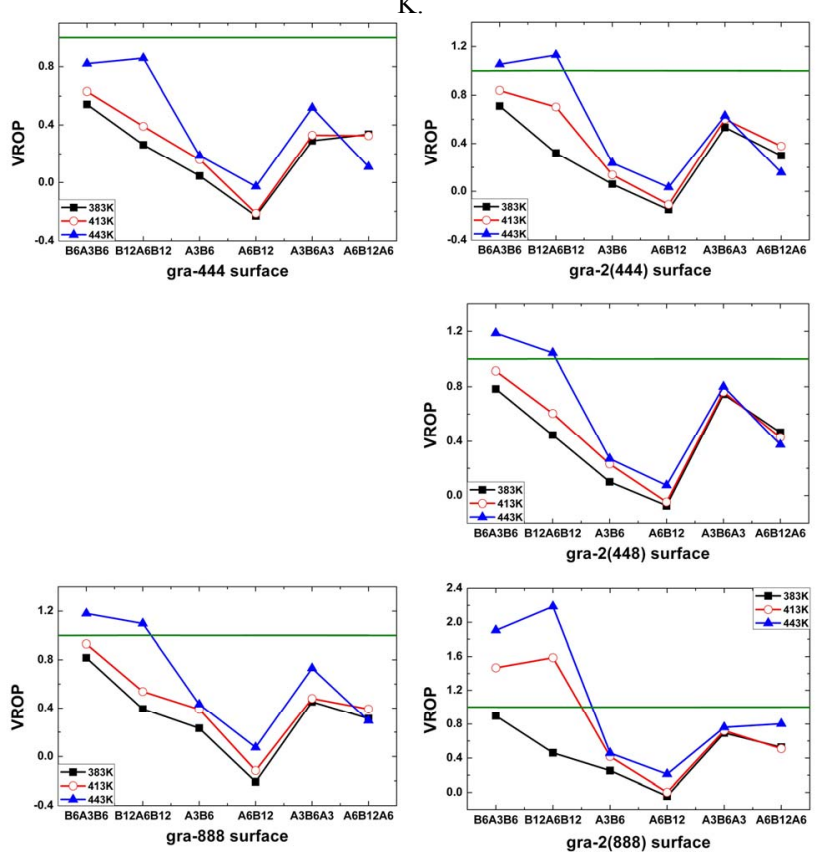

Figure 8 . VROP data for PS-b-PMMA copolymers induced by gra-444, gra-888, gra-2(444), gra-2(448) and gra-2(888) surface at 383, 413 and 443

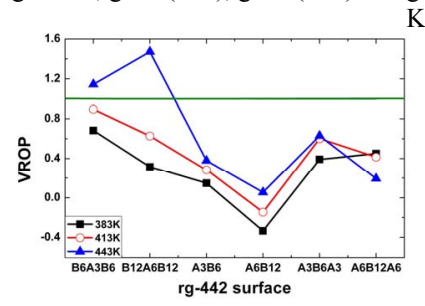
$\mathrm{K}$.
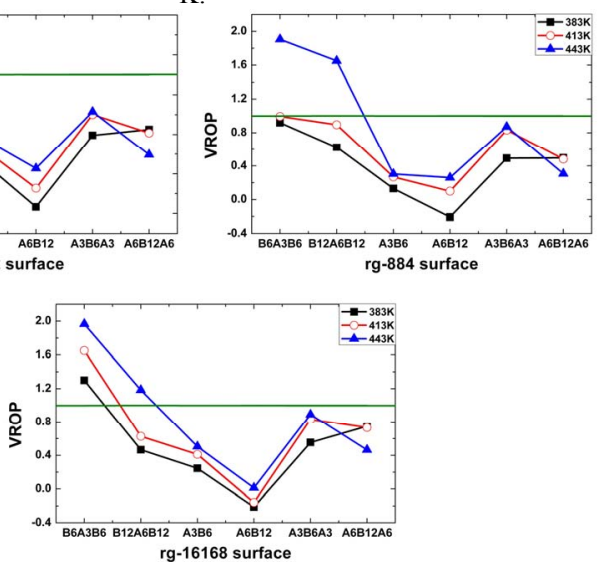

Figure 9. VROP data for PS-b-PMMA copolymers induced by rg-442, rg884 and rg-16168 surface at 383,413 and $443 \mathrm{~K}$.

\section{COMPARABLE PARAMETERS}

The order parameter $\mathrm{P}$ was defined as the volume average of the difference between local density squared and the overall density squared. The larger the value of the order parameter was, the more obvious the phase separation was. A decrease in P indicated better compatibility or miscibility, and the polymer phases mixed more randomly.

\section{RESUlTS AND DisCUSSIONS}

Figure 2, 3, 4 and 5 showed $\mathrm{P}$ of six kinds of PS-bPMMA copolymers with eighteen inducing surface effects. Several features could be seen in these figures as follows:

(1) In Figure 2 showing the "ci" series surfaces, all the six PS-b-PMMA copolymers demonstrated a relationship of PB6A3B6 < PB12A6B12 < PA3B6 < PA6B12 < PA3B6A3 $<$ PA6B12A6, which can be classified into two kinds of main relationship: (a) the first one was $\mathrm{P}$ "BAB" $<\mathrm{P}$ "AB" $<$ $\mathrm{P}$ "ABA". It can be derived when the segment of "A" or " $\mathrm{B}$ " located at both sides in copolymers, there were much more opportunity to "meet" with the same kind of segment, then it was easy to form the microscopic areas consisting of the same component, even lead to a microscopic separation. In addition, the weight percentage values of " $A$ " were $20.87 \%$, $33.99 \%$ and $50.96 \%$ for "BAB", "AB" and "ABA", respectively. They were all resulted from the difference in the copolymer-type. (b) the second one was PB6A3B6 < PB12A6B12, PA3B6 < PA6B12 and PA3B6A3 < PA6B12A6, which could be reduced as Pshort-chained < Plong-chained. It also could be attributed to the structure of copolymers, the longer chain formed larger congregation areas than the shorter ones.

For each kind of copolymers, the $\mathrm{P}$ values at higher temperature was higher than that at lower temperature, which was a relation of $\mathrm{P} 383 \mathrm{~K}<\mathrm{P} 413 \mathrm{~K}<\mathrm{P} 443 \mathrm{~K}$. It revealed that it was likely to form microscopic separation under higher temperature. Deduced from the high $\mathrm{P}$ value at $443 \mathrm{~K}$, compared with it at $383 \mathrm{~K}$ and $413 \mathrm{~K}$ for every copolymer, high temperature could lead the copolymer present microscopic separation easily, especially for the longer chained copolymer of "ABA" group, induced by ci-888 surface, such as A6B12A6 copolymer.

(2) In Figure 3 showing the "co" series surfaces, all the six PS-b-PMMA copolymers demonstrated a relationship the same as it induced by "ci" surfaces. However, the six PS-bPMMA copolymers induced by co-4432 and co- 8832 surfaces presented different from the others.

For the copolymers induced by co-4432 surface, it showed a relationship of PB6A3B6 $<$ PA3B6A3 $<$ PA3B6 $<$ PA6B12 < PB12A6B12 < PA6B12A6. It also could be classified into three relationships: (a) Pshort-chained < Plong-chained for each group, the reason had been mentioned above. (b) For short-chained copolymers, PB6A3B6 < PA3B6A3 < PA3B6, that was, P"BAB" < P"ABA" < P"AB". The co-4432 surface had discontinuous mask distributions, which meant that the polymer did not have enough room to move and spread; they could only adjust themselves in the "small cubic spaces" formed by neighboring column masks, form a microscopic area consisting of the same segments, even form phase separation. Therefore, the longer chained "BAB" and "ABA" group copolymers presented lower $\mathrm{P}$ values. On the contrary, the short chained "AB" group copolymer was more flexible than the longer chained "BAB" and "ABA" to adjust its position, then to form a microscopic area consisting of the same segments, so the $\mathrm{P}$ values of "AB" group presented the highest values. (c) However, for long-chained copolymers, 
PA6B12 < PB12A6B12 < PA6B12A6, that was, P"AB" < $\mathrm{P}$ "BAB" $<\mathrm{P}$ "ABA", which was different from the short chained copolymer. It mainly attributed to the difference in the chain length. "BAB" and "ABA" of long chained copolymers could have much more opportunity to form ordered microscopic area, compared with the short chained copolymers.

For the copolymers induced by co- 8832 surface, it showed extremely high $\mathrm{P}$ value for A6B12A6 copolymer at $383 \mathrm{~K}, 413 \mathrm{~K}$ and $443 \mathrm{~K}$, compared with the other copolymers. The reason was the same as the highest $\mathrm{P}$ values of "ABA" induced by "ci" series surface.

(3) In Figure 4 showing the "gra" series surfaces, all the six PS-b-PMMA copolymers demonstrated a relationship the same as it induced by "ci" surfaces. But there was a little difference between gra-xxx and gra-2(xxx) series surfaces. The differences of $\mathrm{P}$ values at different temperatures induced by gra-Xxx surfaces were a little larger than it induced by gra-2(xxx) surfaces, except the A6B12A6 copolymer induced by gra-2(888) surface. This was the result from the difference in the inducing surface, that was, gra-xxx surfaces were monodirectional asymmetric and gra-2(xxx) surfaces were monodirectional symmetric.

(4) In Figure 5 showing the "rg" series surfaces, all the six PS-b-PMMA copolymers demonstrated a relationship similar as it induced by "gra" surfaces. It can be deduced from the origination of the "rg" series surfaces, which was bidirectional symmetric surfaces, compared with "gra" surfaces.

It was necessary to define a comparable parameter, denoted as VROP, which was the abbreviation of the variation rates of order parameters, to discuss the influencing effect of inducing surfaces. Its definition process was: set the plain PS-b-PMMA copolymers as a reference, and its order parameter values were named as "A"; the order parameter values of PS-b-PMMA copolymers induced by surfaces were named as "B", then the values of (B-A)/A were the results of VROP. By comparing the VROP values, we could detect which inducing surface was the effective inducing surfaces on changing the phase morphology of the PS-b-PMMA copolymers.

Figure $6,7,8$ and 9 showed VROP values of six kinds of PS-b-PMMA copolymers with eighteen inducing surface effects mentioned above, based on the plain copolymers. The VROP values can be applied to illuminate the extent of inducing effect on changing the phase morphology of PS-bPMMA copolymers with eighteen surfaces induced. A reference line was drawn through the VROP value of 1 in these four figures; when an VROP value lay above the line, the inducing effect was considered a reinforcing immiscible effect; otherwise, the inducing effect was considered a weakening immiscible effect, in addition, the VROP values below zero were considered reinforcing miscible effect. Such reinforcing immiscible and reinforcing miscible effect of six PS-b-PMMA copolymers induced by each inducing surface at 383, 413 and $443 \mathrm{~K}$ was listed in Table III in details. There were several striking features of these data in Table III, combined with Figure 6, 7, 8 and 9:
(1) The trends seen in these four figures were similar, except it induced by co-4432 and co-8832. It can be deduced from the different structure between these two (co-4432 and co-8832) and the others surfaces. The "ci", "co", "gra" and "rg" surfaces except co-4432 and co-8832 had continuous mask distributions, similar as the situation of its plain copolymers without surface induced, which allowed the PSb-PMMA copolymers to move and spread more freely until they could adjust themselves to reach stable phase separation. In contrast, the co-4432 and co-8832 had discontinuous mask distributions, which made the copolymer do not have enough room to move and spread; they could only adjust themselves in the "small cubic spaces" formed by neighboring column masks. Therefore, the VROP values of copolymers with co4432 and co-8832 surfaces induced were all above 1 at 383 , 413 and $443 \mathrm{~K}$, furthermore, their values were one order of magnitude higher than the values of other surfaces, which meant such two surfaces played an extreme reinforcing immiscible effect.

(2) The number of the case above 1 at 383, 413 and 443 $\mathrm{K}$ was 22,28 and 41, respectively, which was in rising trend; whereas the number of the case below zero at 383,413 and $443 \mathrm{~K}$ was 15,13 and 4, respectively, which was in decreasing trend. It meant the temperature played important role in changing the degrees of phase separation. It can be deduced mainly from the big difference between the diffusion abilities of PS and PMMA segments40.

(3) The blank of "cases above 1" in the table meant the surface played a small role in inducing phase separation, that was, ci-444, ci-882, co-444, co-448, co-884, gra-444, gra888, gra-2(444), gra-2(448), gra-2(888), rg-442 and rg-884 at $383 \mathrm{~K}$; ci-444, ci-882, co-444, gra-444, gra-888, gra-2(444), gra-2(448), rg-442 and rg-884 at $413 \mathrm{~K}$; ci-882, co-444, gra444 at $443 \mathrm{~K}$. Among them, the VROP values of ci-882, co444 and gra-444 surfaces were all below 1 at 383, 413 and $443 \mathrm{~K}$, this meant such three surfaces had little effect on changing the phase morphology of copolymers. It was the result from their relative smooth surface that led them to present little inducing effects.

\section{SUMMARIES}

Mesoscopic simulations were carried out on PS-bPMMA copolymers with surfaces factored in. The simulation results showed that four series surfaces presented different effect on improving the degree of order in the microscopic phases, especially effective on the "ABA" groups copolymers, "AB" groups the second and "BAB" groups the last. In addition, the values of order parameter of the long chains were higher than those of the short ones.

The designed "ci", "co", "gra" and "rg" except co-4432 and co-8832 surfaces had continuous mask distributions, which are different from co-4432 and co-8832 surfaces, which had discontinuous mask distributions. Therefore, no matter which temperature was considered, the surfaces had a stronger influence on changing the phase morphology of copolymers, especially the co-4432 and co- 8832 surfaces, which performed the most intensive extent of inducing effect on changing the phase morphology of PS-b-PMMA copolymers. Higher temperature showed a more obvious 
effect on changing the phase morphology, but it was not an effective factor on changing the $\mathrm{P}$ values.

\section{ACKNOWLEDGMENTS}

This work is supported by Promotive Research Fund for Excellent Young and Middle-aged Scientists of Shandong Province (BS2010CL048), Shandong Province Higher School Science \& Technology Fund Planning Project (J10LA61) and Zaozhuang Scientific and Technological Project (200924-2). In addition, thanks for the support of the state key laboratory of theoretical and computational chemistry on the softwares.

\section{REFERENCES}

[1] Stupp S.I.. Self-assembly of rodcoil molecules. Current Opinion in Colloid \& Interface Science. [J], 1998, 3, PP20-26.

[2] Mao G.; Ober C.K.. Handbook of Liquid Crystals. Vol 3, Wiley-VCH, Weinheim, 1998.
[3] Gallot B.. Comb-like and block liquid crystalline polymers for biological applications. Progress in Polymer Science. [J], 1996, 21(6), PP1035-1088.

[4] Loos K.; Munoz-Guerra S.. Supermolecular Polymers, Dekker, New York, 2000.

[5] Kim K.H.; Huh J.; Jo W.H.. Wirelike Micelle Formed by a T-Shaped Graft Copolymer with a Rigid Backbone. Macromolecules [J], 2004, 37, PP676-679.

[6] Hamley I.W.. Nanostructure fabrication using block copolymers. Nanotechnology [J], 2003, 14, PPR39-R54.

[7] Hamley I.W.. The Physics of Block Copolymers. Oxford University Press, Oxford, 1998.

[8] Bucknall D.G.; Anderson H.L.. Polymers Get Organized. Science [J], 2003, 302, PP1904-1905.

[9] Matsen M.W.; Schick M.. Stable and unstable phases of a diblock copoymer melt. Physical Review Letters. [J], 1994, 72, PP2660-2663.

[10] Chen J.T.; Thomas E.L.; Ober C.K.; Mao G.. Self-Assembled Smectic Phases in Rod-Coil Block Copoymers. Science [J], 1996, 273, PP343-346.

TABLE I. DETAILS OF THE DESIGNED PS-B-PMMA COPOLYMERS MODELS

\begin{tabular}{|l|l|l|c|c|c|}
\hline $\begin{array}{c}\text { Classified } \\
\text { group }\end{array}$ & \multicolumn{1}{|c|}{ System } & $\begin{array}{c}\text { wt\% of PMMA } \\
\text { content }\end{array}$ & $\begin{array}{c}\text { IPMa } \\
\mathbf{( 3 8 3 K )}\end{array}$ & $\begin{array}{c}\text { IPMa } \\
\mathbf{( 4 1 3 K )}\end{array}$ & $\begin{array}{c}\text { IPMa } \\
\mathbf{( 4 4 3 K )}\end{array}$ \\
\hline \multirow{2}{*}{ "AB" group } & $\mathrm{A} 3 \mathrm{~B} 6$ & 66.01 & 0.92057 & 0.91268 & 1.02383 \\
\cline { 2 - 6 } & $\mathrm{A} 6 \mathrm{~B} 12$ & 66.01 & 0.92057 & 0.91268 & 1.02383 \\
\hline \multirow{2}{*}{$\begin{array}{c}\text { "ABA" } \\
\text { group }\end{array}$} & $\mathrm{A} 3 \mathrm{~B} 6 \mathrm{~A} 3$ & 49.04 & 0.59536 & 0.74319 & 0.80883 \\
\cline { 2 - 6 } & $\mathrm{A} 6 \mathrm{~B} 12 \mathrm{~A} 6$ & 49.04 & 0.59536 & 0.74319 & 0.80883 \\
\hline $\begin{array}{l}\text { "BAB" } \\
\text { group }\end{array}$ & $\mathrm{B} 6 \mathrm{~A} 3 \mathrm{~B} 6$ & 79.13 & 1.07334 & 1.15849 & 1.13485 \\
\cline { 2 - 6 } & $\mathrm{B} 12 \mathrm{~A} 6 \mathrm{~B} 12$ & 79.13 & 1.07334 & 1.15849 & 1.13485 \\
\hline
\end{tabular}

TABLE II. INFORMATION OF “CI”, “CO”, “GRA” AND “RG” SERIES SURFACES

\begin{tabular}{|c|c|c|c|c|c|}
\hline Type & $\begin{array}{l}\text { Included } \\
\text { Surfaces }\end{array}$ & Example & Scheme & Mask Type & Name Explanation \\
\hline ci-xxx & ci- 444 , ci- 882 & ci-444 & S1 & semicircular balls & $\begin{array}{l}\text { four semicircular balls in } \\
\text { four surface sides, the } \\
\text { radius is } 4 \mathrm{~nm}\end{array}$ \\
\hline $\operatorname{co}-4 x x$ & $\begin{array}{l}\text { co- } 444, \text { co- } 448, \\
\text { co- } 4412, \quad \text { co- } \\
4432\end{array}$ & co-444 & S2 & $\begin{array}{l}\text { equal spaced } \\
\text { cubic columns }\end{array}$ & $\begin{array}{l}\text { divided by equal space } \\
\text { into four parts to form } \\
\text { two columns in four } \\
\text { surface sides, the height } \\
\text { is } 4 \mathrm{~nm}\end{array}$ \\
\hline co- $8 \mathrm{xx}$ & $\begin{array}{l}\text { co- } 884, \text { co- } 888, \\
\text { co- } 8812, \quad \text { co- } \\
8832\end{array}$ & co- 884 & S3 & $\begin{array}{l}\text { equal spaced } \\
\text { cubic columns }\end{array}$ & $\begin{array}{l}\text { divided by equal space } \\
\text { into eight parts to form } \\
\text { four columns in four } \\
\text { surface sides, the height } \\
\text { is } 4 \mathrm{~nm}\end{array}$ \\
\hline gra-xxx & $\begin{array}{l}\text { gra- } 444, \\
\text { gra- } 888\end{array}$ & gra-444 & S4 & $\begin{array}{l}\text { the same widths, } \\
\text { gradually } \\
\text { increasing height } \\
\text { columns, } \\
\text { monodirectional } \\
\text { asymmetric } \\
\end{array}$ & $\begin{array}{l}\text { divided by equal space } \\
\text { into four parts in face-to- } \\
\text { face sides to four small } \\
\text { surfaces with gradually } \\
\text { increasing height, the } \\
\text { highest height is } 4 \mathrm{~nm}\end{array}$ \\
\hline $\begin{array}{l}\text { gra- } \\
2(x x x)\end{array}$ & $\begin{array}{ll}\text { gra-2(444), } & \text { gra- } \\
2(448), & \text { gra- } \\
2(888) & \end{array}$ & gra-2(444) & S5 & $\begin{array}{l}\text { the same as gra- } \\
\mathrm{xxx} \text { type, but } \\
\text { monodirectionl } \\
\text { symmetric }\end{array}$ & $\begin{array}{l}\text { with the same two } \\
\text { symmetric parts as gra- } \\
444 \text { surface }\end{array}$ \\
\hline rg-xxx & $\begin{array}{l}\text { rg-442, rg-884, } \\
\text { rg-16168 }\end{array}$ & rg-442 & S6 & $\begin{array}{l}\text { similar as gra-xxx } \\
\text { type, but } \\
\text { bidirectional } \\
\text { symmetric }\end{array}$ & $\begin{array}{l}\text { the highest height of both } \\
\text { face-to-face sides is } 4 \\
\mathrm{~nm} \text {, the divided surface in } \\
\text { one semi-section is two } \\
\text { part }\end{array}$ \\
\hline
\end{tabular}

